\title{
CRISPR screening identifies M1AP as a new MYC regulator with a promoter-reporter system
}

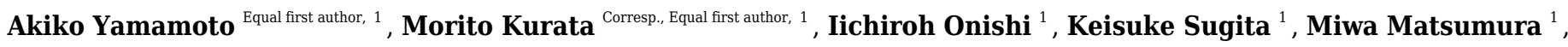 \\ Sachiko Ishibashi ${ }^{1}$, Masumi Ikeda ${ }^{1}$, Kouhei Yamamoto ${ }^{1}$, Masanobu Kitagawa ${ }^{1}$ \\ 1 Department of Comprehensive Pathology, Graduate School of Medical and Dental Sciences, Tokyo Medical and Dental University, Tokyo, Japan \\ Corresponding Author: Morito Kurata \\ Email address: kurata.pth2@tmd.ac.jp
}

Background. MYC is one of the proto-oncogenes contributing to tumorigenesis in many human cancers. Although the mechanism of MYC regulation is still not fully understood, learning about the comprehensive mechanism controlling the transcriptional activity of MYC will lead to therapeutic targets. The CRISPR/Cas9 library system is a simple and powerful screening technique. This study aims to identify new transcriptional upstream activators of MYC using the CRISPR activation library with new promoterreporter systems.

Methods and Results. The MYC promoter-reporter system was developed with a photoconvertible fluorescent protein, Dendra2, and named "pMYC-promoter-Dendra2." This MYC promoter-reporter system was designed to harbor a proximal MYC promoter at $-3.1 \mathrm{~kb}$. Both the CRISPR activation library and pMYC-promoter-Dendra2 were induced to HEK 293T cells, and Dendra2-positive cells, that are supposed that MYC should be upregulated, were collected individually by a cell sorter. Among the 169 cells collected, 12 clones were successfully established. Then, pMYC-promoter-Dendra2 was transfected again into these 12 clones, and 2 of 12 clones showed Dendra 2 positivity. In this procedure, the cells with nonspecific autofluorescence were correctly distinguished by utilizing the photoswitchable character of Dendra2. Using extracted guide DNA of these two Dendra2 positive clones, polymerase chain reaction (PCR) was performed to amplify the guide RNA (gRNA) containing region, which was introduced by the CRISPR activation library. Eventually, PLEKHO2, MICU, MBTPS1, and M1AP were identified, and these gRNAs were transfected individually into HEK 293T cells again using the CRISPR activation system. Only MIAP gRNA transfected cells showed Dendra2-positive fluorescence. Then, the overexpression vector for MIAP with a doxycycline-inducible vector confirmed that MIAP induced high MYC expression by real-time quantitative PCR and western blot. Furthermore, the dual-luciferase assay showed a significant increase of promoter activity, and MYC mRNA was higher in M1AP- overexpressing cells. M1AP is highly expressed in several cancers, though, a positive correlation between M1AP and MYC was observed only in human acute myeloid leukemia.

Conclusion. The present study confirmed that the experimental method using the CRISPR library technology functions effectively for the identification of molecules that activate endogenous MYC. This method will help elucidate the regulatory mechanism of MYC expression, as well as supporting further drug research against malignant tumors. 


\section{CRISPR Screening Identifies M1AP as a New MYC}

\section{Regulator with a Promoter-Reporter System}

4

5 Akiko Yamamoto ${ }^{1,2}$, Morito Kurata ${ }^{1,2 * *}$, Iichiroh Onishi ${ }^{1}$, Keisuke Sugita ${ }^{1}$, Miwa

6 Matsumura $^{1}$, Sachiko Ishibashi ${ }^{1}$, Masumi Ikeda ${ }^{1}$, Kohei Yamamoto ${ }^{1}$, Masanobu Kitagawa ${ }^{1}$

7

8 1. Department of Comprehensive Pathology, Graduate School of Medical and Dental Sciences,

9 Tokyo Medical and Dental University, Tokyo, JAPAN

10 2. These authors contributed equally to this work.

11

12

**Corresponding Author:

Morito Kurata,

1-5-45 Yushima, Bunkyo-ku, Tokyo 113-8510, JAPAN

E-mail address: kurata.pth2@tmd.ac.jp

17

8

(2)

1




\section{Abstract}

27 Background. $M Y C$ is one of the proto-oncogenes contributing to tumorigenesis in many human cancers. Although the mechanism of $M Y C$ regulation is still not fully understood, learning about the comprehensive mechanism controlling the transcriptional activity of $M Y C$ will lead to therapeutic targets. The CRISPR/Cas9 library system is a simple and powerful screening technique. This study aims to identify new transcriptional upstream activators of $M Y C$ using the CRISPR activation library with new promoter-reporter systems.

Methods and Results. The $M Y C$ promoter-reporter system was developed with a photoconvertible fluorescent protein, Dendra2, and named "pMYC-promoter-Dendra2." This $M Y C$ promoter-reporter system was designed to harbor a proximal $M Y C$ promoter at $-3.1 \mathrm{~kb}$. Both the CRISPR activation library and pMYC-promoter-Dendra2 were induced to HEK 293T cells, and Dendra2-positive cells, that are supposed that $M Y C$ should be upregulated, were collected individually by a cell sorter. Among the 169 cells collected, 12 clones were successfully established. Then, $\mathrm{p} M Y C$-promoter-Dendra 2 was transfected again into these 12 clones, and 2 of 12 clones showed Dendra 2 positivity. In this procedure, the cells with nonspecific autofluorescence were correctly distinguished by utilizing the photoswitchable character of Dendra2. Using extracted guide DNA of these two Dendra2 positive clones, polymerase chain reaction (PCR) was performed to amplify the guide RNA (gRNA) containing region, which was introduced by the CRISPR activation library. Eventually, PLEKHO2, MICU, MBTPS1, and $M 1 A P$ were identified, and these gRNAs were transfected individually into HEK 293T cells again using the CRISPR activation system. Only MIAP gRNA transfected cells showed

47 Dendra2-positive fluorescence. Then, the overexpression vector for $M 1 A P$ with a doxycyclineinducible vector confirmed that $M I A P$ induced high $M Y C$ expression by real-time quantitative 
49 PCR and western blot. Furthermore, the dual-luciferase assay showed a significant increase of 50 promoter activity, and $M Y C$ mRNA was higher in $M I A P$ - overexpressing cells.

$51 M 1 A P$ is highly expressed in several cancers, though, a positive correlation between $M 1 A P$ and

$52 M Y C$ was observed only in human acute myeloid leukemia.

53 Conclusion. The present study confirmed that the experimental method using the CRISPR

54 library technology functions effectively for the identification of molecules that activate

55 endogenous $M Y C$. This method will help elucidate the regulatory mechanism of $M Y C$ expression, 56 as well as supporting further drug research against malignant tumors.

57

58

59

60

61

62

\section{Introduction}

$M Y C$ is one of the most well-known proto-oncogenes, contributing to tumorigenesis in many human cancers. It can function as a transcriptional factor in both cancerous and non-cancerous cells, and it is involved in cell growth, proliferation, metabolism, and transformation (Dang, 2012). The Wnt- $\beta$-catenin signaling pathway is one of the most well-described pathways involved in the regulation of MYC. Due to mutations in the adenomatous polyposis coli (APC) gene or abnormalities in glycogen synthase kinase-3 $\beta$, Wnt-signal is stimulated and the excessive accumulation of $\beta$-catenin occurs. Then, active $\beta$-catenin moves into the nucleus and binds to $\mathrm{T}$ cell factor (TCF), followed by inducing MYC expression (Liu et al., 2002) (Novak, Dedhar, 1999). Mitogen-activated protein kinase (MAPK), which is a serine/threonine kinase, and its subfamily extracellular signal-regulated kinase (ERK), regulate $M Y C$ transcriptional activity by inducing phosphorylation of MYC (MAPK/ERK signaling pathway). Furthermore, as noncoding RNA, microRNAs like miR-34 (Kong et al., 2008) and let-7 (Sampson et al., 2007) suppress $M Y C$ expression by binding to the 3'-untranslated region of $M Y C$ mRNA. As for long 
72 noncoding RNAs, PVT1 regulates the expression of $M Y C$ by inhibiting the degradation of MYC

73 protein (Tseng et al., 2014). Thus, it has been reported that various regulatory mechanisms

74 function in a complex fashion regarding $M Y C$ expression. Though, other molecules and elements

75 like crosstalk between each pathway are not fully understood.

76 Concerning the high $M Y C$ expression rate of multiple malignant tumors, elucidating the

77 mechanism controlling the transcriptional activity of $M Y C$ is a crucial issue because it will lead

78 to therapeutic targets. Using the CRISPR/Cas9 library system, with its collection of more than

79 70,290 different guide RNAs (gRNAs), enables us to identify molecules that relate knockout or

80 activation of endogenous genes. To date, the CRISPR library has been utilized for various

81 studies. Shalem et al. (2014) identified genes whose loss is involved in resistance to vemurafenib

82 by introducing the knockout library into a human melanoma cell line followed by treatment with

83 BRAF inhibitor vemurafenib. In contrast, Konnermann et al. (2015) identified molecules that

84 exhibit resistance to PLX-4720 by introducing an activation library. In this way, the CRISPR

85 library has been used to identify the certain genes involved in drug sensitivity or resistance, toxin

86 resistance, and hypoxia response (Konermann et al., 2015; Gilbert et al., 2014; Kampmann,

87 2018; Kurata et al., 2018; Shalem et al., 2014; Wang et al., 2014; Koike-Yusa et al., 2014;

88 Doench et al., 2016; Jain et al., 2016); however, a screening of molecules that act on the

89 promoter region of a specific gene and control transcriptional activity has not yet been tested. In

90 addition, autofluorescence is highly problematic for collecting fluorescent cells in the screening.

91 Therefore, a photoconversion protein, Dendra2, was used in this system. Dendra2 is derived

92 from the octocoral Dendronephthya sp. T. and can change color with UV-violet light (405 nm

93 laser). This study aims to utilize the CRISPR activation library for the detection of new 
94 transcriptional activators of $M Y C$. For this purpose, we developed an original tool for evaluating

95 MYC activity and establishing screening methods.

96

97 Materials \& Methods

MYC Promoter-Reporter System and Lentivirus Plasmids

99 The Polymerase chain reaction (PCR) primers were designed across the proximal MYC

100 promoter at $-3.1 \mathrm{~kb}$ from the MYC transcriptional start site (TSS), and PCR was performed with

101 KOD-FX (Toyobo). The primers were 5'-TTAGCTAGCGAGGGTTTTCTTTGAGGGGC-3'

102 (forward) and 5'-AACGGATCCCGGAGATTAGCGAGAGAGGA-3' (reverse).

103 A plasmid of Dendra2 (pDendra2) was purchased from Takara-Clontech. The

104 cytomegalovirus immediate early promoter (PCMVIE) was removed with restriction enzymes

105 (Afl III and Nhe I), and the multi-cloning site was replaced with Nhe I and BamH I. Amplified

106 fragments of $-3.1 \mathrm{~kb}$ of the $M Y C$ promoter region with Nhe I and BamH I restriction enzyme

107 sites were integrated into pDendra2. The new reporter system with Dendra2 and the $-3.1 \mathrm{~kb} M Y C$

108 promoter region (pMYC-promoter-Dendra2) was used for further experiments. The double-

109 stranded DNA fragments of $M 1 A P$ with attB sites were purchased from gBlocks ${ }^{\circledR}$ (IDT) and

110 generated into the pENTR221 vector using Gateway ${ }^{\circledR}$ BP clonase. Then they were transferred to

111 the TripZ-TRE-DEST-IRES-GFP-EF1A-rtTA vector (Kurata et al., 2016) using Gateway® LR

112 clonase.

113

114 Cell Culture and SAM Library Screening

115 Human embryonic kidney cells (HEK 293T) were maintained in Dulbecco’s modified Eagle’s

116 medium (DMEM, Fuji Film) with 10\% fetal bovine serum and penicillin/streptomycin with 5\% 
$117 \mathrm{CO}_{2}$ at $37^{\circ} \mathrm{C}$ as previously used (Kurata et al., 2016). To transduce the lentivirus, HEK 293T

118 cells $\left(5 \times 10^{5}\right.$ cells/well $)$ were seeded in a six-well cell culture plate one day before the

119 transfection. The next day, $3 \mu \mathrm{g}$ of lentivirus plasmid was transfected with $1 \mu \mathrm{g}$ of $\mathrm{pMDG}$ and 2

$120 \mu \mathrm{g}$ of pCMV using Lipofectamine ${ }^{\mathrm{TM}} 3000$ Reagent (Invitrogen) according to previous work

121 (Kurata et al., 2016). Twelve hours after transfection, the medium was changed to fresh DMEM.

122 The virus supernatant was harvested at $48 \mathrm{~h}$ after transfection, then filtered with Millex ${ }^{\mathrm{TM}}-\mathrm{HP}$

$1230.45 \mu \mathrm{m}$ (Millipore). The target HEK 293T cells $\left(5 \times 10^{5}\right.$ cells/well) were seeded in a six-well

124 plate one day prior to transduction and were transduced with this lentivirus supernatant with 5

$125 \mu \mathrm{g} / \mathrm{ml}$ polybrene (Sigma). The used lentiviral plasmids were lentiMPHv2 (Addgene, \#89308),

126 lentidCAS-VP64_blast (Addgene, \#61425), and lentiSAMv2 (Addgene, \#61597). At first,

127 lentiMPHv2 was transduced into HEK 293T and treated with Hygromycin B for 2 weeks. Then,

128 lentidCAS9-VP64 was induced in MPH-expressing HEK 293T cells and treated with Blasticidin

129 for 2 weeks. The MPH-dCas9-VP64-expressing HEK 293T applied to further experiments. The

130 control and MYC activation gRNA (Fig. S1C) were induced in MPH-dCas9-VP64-expressing

131 HEK 293T cells. The CRISPR activation library, lentiSAMv2, was also transduced into MPH-

132 dCas9-VP64-expressing HEK 293T cells and then treated with Zeocin $(300 \mu \mathrm{g} / \mathrm{ml}$, Invivogen $)$

133 for 2 weeks.

134

135 Collection of Dendra2-Positive Cells and Identification of gRNAs

136 After 2 weeks of selection with Zeocin, $\mathrm{p} M Y C$-promoter-Dendra2 was transfected into SAM

137 library lentivirus-transduced cells with Lipofectamine ${ }^{\mathrm{TM}} 3000$ Reagent (Invitrogen). HEK 293T

138 cells transfected with pMYC-promoter-Dendra2 were evaluated for Dendra2-positive cells by

139 fluorescence microscope (Keyence). Cells were collected at $72 \mathrm{~h}$ and washed with phosphate- 
140 buffered saline (PBS) and applied to the cell sorter. The Dendra2-positive cells were collected

141 into a 96-well plate with MoFlo XDP (Beckman Coulter).

142 A total of 169 cells were collected, and 12 clones were successfully established. Collected

143 clones were centrifuged and washed with PBS solution and pelletized. Lysis buffer solution with

144 proteinase $\mathrm{K}(20 \mathrm{mg} / \mathrm{ml})$ was added to cells and left for $30 \mathrm{~min}$ at $60^{\circ} \mathrm{C}$. After treatment at $94^{\circ} \mathrm{C}$

145 for $10 \mathrm{~min}$, the lysate was centrifuged for $15 \mathrm{~min}$, and the supernatant was collected as genome

146 DNA (gDNA) containing fractions. Using extracted gDNA of each collected clone, PCR was

147 conducted with GoTaq ${ }^{\circledR}$ qPCR Master Mix (Promega) with the following primers: 5'-

148 GAGGGCCTATTTCCCATGAT -3' (forward) and 5'-GAGGGCCTATTTCCCATGAT-3'

149 (reverse). PCR product containing the gRNA region was employed for TA cloning with

150 pGEM®-T Vector Systems (Promega) and gRNAs were sequenced by Sanger-sequencing.

151

152 Preparation of CRISPR backbone plasmid with target gRNAs and Transcriptional activation 153 for MYC using the CRISPR Activation System

154 The oligonucleotides for each target gRNA sequence were designed using the list from the 155 SAM library and integrated into the pENTR221/U6/stuffer/MS2 vector (Kurata et al., 2018).

156 The MPH-dCas9-VP64-expressing HEK 293T cells ( 1 x $10^{5}$ cells $\left./ \mathrm{ml}\right)$ were seeded in a $24-w e l l$ 157 cell culture plate. The next day, $2.5 \mu \mathrm{g}$ of individual gRNA vector and $\mathrm{p} M Y C$-promoter-Dendra2 158 were transfected with Lipofectamine ${ }^{\mathrm{TM}} 3000$ Reagent. At 48 hours after the transfection, 159 the $M Y C$ transcriptional activation was evaluated by Dendra2-positivity by Keyence. 160

\section{Evaluation of MYC expression}


162

163

164

165

166

167

168

169

170

171

172

173

174

175

176

177

178

179

180

181

182

183

184

A pTRIPZ-MIAP-GFP was transduced into the HEK 293T cells. These transduced cells were selected with puromycin $(1 \mu \mathrm{g} / \mathrm{ml}$, Invitrogen) for 2 weeks. Seventy-two hours after the induction of Doxycycline (DOX) $(1 \mu \mathrm{g} / \mathrm{ml})$, the collected cells were employed for RNA isolation.

RNA was isolated using an RNeasyR Mini Kit (Qiagen) according to the manufacturer's instructions. Complementary DNA (cDNA) was generated from RNA with ReverTra AceR qPCR RT Master Mix (Toyobo). Beta-ACTIN was used as an endogenous control. Using the ABI Prism 7900HT (Applied Biosystems), quantitative PCR (qPCR) analysis was performed to quantify the RNA level using SYBR Mix. The sequences for the PCR primers used in gene expression were as follows: MYC: 5'-CGACTCTGAGGAGGAACAAGAA-3' (forward) and 5'CAGCAGAAGGTGATCCAGACT-3' (reverse), $\beta$-ACTIN: 5'-CACAGAGCCTCGCCTTTGCC3' (forward) and 5'-CACAGAGCCTCGCCTTTGCC-3' (reverse). The mRNA level of the targeted gene was analyzed by comparison with the standard calibration curve. Western blot analysis was performed according to standard procedures(Kurata et al., 2016). The primary antibodies used were anti-c-Myc (Abcam ab32072) and anti-GAPDH (Santa Cruz sc47724), each at a dilution of 1:1000. The primary antibodies were detected with horseradish peroxidase (HRP)-conjugated secondary antibodies at 1:50000. Protein bands were visualized with Clarity $^{\mathrm{TM}}$ Western ECL Substrate (Bio-Rad).

\section{Dual-Luciferase Reporter Assay}

The fragments of the MYC promoter was integrated into a pGL4 vector (Promega). A pT3.5CAG-M1AP was generated with pENTR221-M1AP and pT3.5-CAG-DEST (Kurata et al., 2018) using Gateway ${ }^{\circledR}$ LR clonase. The luciferase reporter vectors, the pT3.5-CAG-M1AP 
185 vector, and the pRL Renilla luciferase reporter vector were co-transfected in the HEK 293T cells.

186 The samples were harvested 48 and 72 hours after the transfection. Each assay was biologically

187 triplicated and repeated. Luciferase activities were measured using the dual-luciferase reporter

188 assay system (Promega) and Lumat LB9507 (Perkin Elmer). RNA was harvested at the same

189 time points and the expression of $M Y C$ mRNA was analyzed by qPCR.

190

191 M1AP and MYC expressions in databases

192 For the purpose of comparing gene expression between cancer and non-cancerous tissue, the

193 GEPIA web server was employed (Tang et al., 2017) (http://gepia2.cancer-pku.cn/).

194 Datasets for AML samples were used in BloodSpot database, that database was used to retrieve

195 gene expression data from curated human and murine microarray and RNA-sequencing (RNA-

196 Seq) datasets (Bagger et al., 2016). GSE42519,

197 GSE13159, GSE15434, GSE61804, GSE14468, and The Cancer Genome Atlas (TCGA) datasets

198 were used for analysis. In addition, following database were used; The Human Protein Atlas

199 (https://www.proteinatlas.org/) and cBioPortal (https://www.cbioportal.org) using TCGA data

200 (Cancer Genome Atlas Research Network et al., 2013; Gao et al., 2013; Cerami et al., 2012)

201

202 Statistical analysis

203 The data were statistically analyzed using the EZR version 1.36 software. A value of $p<0.05$

204 was considered statistically significant for all analyses.

205

206 Results

207 Development of the MYC Promoter-Reporter System for Library Screening 
208 Using PCR primers designed across the proximal MYC promoter at $-3.1 \mathrm{~kb}(-3134$ to -40$)$

209 from the MYC TSS, PCR was performed. The MYC promoter region was integrated upstream of

210 Dendra2. The pDendra2 harboring the $3.1 \mathrm{~kb} M Y C$ promoter region was called "pMYC-

211 promoter-Dendra2" for further experiments (Fig. 1SA). A plasmid pMYC-promoter-Dendra2 and 212 gRNA of MYC and HPRT (as a negative control) were transfected in HEK 293T cells using the 213 CRISPR activation system. We confirmed that Dendra2 showed positivity only in the cells with 214 gRNA for $M Y C$ (Fig. 1SB, C). Therefore, pMYC-promoter-Dendra2 was utilized as a $M Y C$ 215 promoter-reporter analysis tool in this study.

216

217

218

219

220

221

222

223

224

225

226

227

228

229

230

231

\section{MYC Transcriptional Activator Screening Using the CRISPR Activation Library}

To screen transcriptional activators working on the $M Y C$ promoter region, the CRISPR activation library was employed. The SAM library lentivirus was infected into MPH-dCas9VP64-expressing HEK 293T cells (1 X 107 cells). After selection, pMYC-promoter-Dendra2 was transfected into these HEK 293T cells. The multiplicity of infection (MOI) ranged from 1.4 to

\section{2 .}

At $72 \mathrm{~h}$ after transfection, cells were proceeded to Dendra2-positive cell collection using flow cytometry (positive ratio: $0.017 \%$ ). Sorted single cells were collected into a 96-well plate and incubated for 2 weeks. Among the collected 169 cells, 12 clones were successfully established (Fig. 1).

\section{Second Screening to Identify Candidate Genes as MYC Activators}

For the second screening, pMYC-promoter-Dendra2 was again transfected into the 12 clones. Using fluorescence microscope, clones 3 and 5 were shown to be truly Dendra2 positive (Fig. 2A-L), and the photoswitchable attribute of Dendra2 was useful for distinguishing it from cells 
232 with non-specific autofluorescence (Fig. 2S). Using extracted gDNA of clones 3 and 5, PCR was

233 performed to amplify the gRNA containing region, which was introduced by the CRISPR

234 activation library. Then, the PCR product was employed for TA cloning, followed by colony

235 PCR. By this method, four candidate genes-PLEKHO2, MICU, MBTPS1, and MIAP were

236 identified (Table 1).

237 Each candidate gRNA was transfected to the HEK 293T cells using the CRISPR activation 238 system accompanied by $\mathrm{p} M Y C$-promoter-Dendra2. Because Dendra2 was positive only in the 239 cells transfected with MIAP gRNA (Fig. 3A-D), MIAP was considered as the most promising 240 gene that would increase $M Y C$ transcriptional activity. Then, double-stranded DNA fragments 241 coding the full length of $M I A P$ induced into Doxycycline-inducible lentiviral vector (pTRIPZ$242 M 1 A P-G F P$, Fig. 4A). The pTRIPZ-MIAP-GFP was introduced into HEK 293T cells. Seventy243 two hours after the induction MIAP with DOX $(1 \mu \mathrm{g} / \mathrm{ml})$, high $M Y C$ expression was observed in 244 DOX-induced cells by RT qPCR ( $t$-test, $p<0.05$, Fig. 4B). Furthermore, in the western blots, 245 an increase of MYC expression was also observed in DOX-induced cells (Fig. 4C).

246 Additionally, a dual-luciferase assay conducted with a reporter containing the $M Y C$ promoter 247 region showed a significant increase of promoter activity in $M 1 A P$-overexpressing cells at both 24848 and 72 hours (Fig. 4D and 3S). On the other hand, the MYC mRNA, which was isolated at the 249 same time points, showed mildly higher in $M 1 A P$-overexpressing cells at 48 hours but not at 72 250 hours (Fig. 4E and 3S). Taken together, although MIAP was confirmed to activate the MYC 251 promoter strongly, the MYC mRNA itself might be regulated also by other factors.

252 
According to the GEPIA web server (Tang Z et al., 2017) (http://gepia2.cancer-pku.cn/),

255 high $M 1 A P$ expression is observed in cervical carcinoma, acute myeloid leukemia (AML),

256 ovarian carcinoma, thyroid carcinoma, uterine endometrial carcinoma, and uterine

257 carcinosarcoma when compared to normal tissue (Fig. 4S A). Among these carcinomas, a

258 correlation analysis using cBioPortal revealed a mild positive correlation between $M Y C$ and

259 MIAP only in AML (Fig. 5A, Fig. 4S B-F). Therefore, we focused on hematopoietic cells, and

$260 M 1 A P$ expression was analyzed using the BloodSpot database. As a result, $M 1 A P$ was

261 significantly higher in AML tissue compared to normal bone marrow tissue (Fig. 5B).

262 Interestingly, MIAP was highly expressed in AML, especially with t (8;21) (Fig. 5C).

263

\section{Discussion}

265 In this study, we created a new reporter vector that incorporates the $M Y C$ promoter region

266 across the proximal $-3.1 \mathrm{~kb}$ from the TSS and photoconversion fluorescent protein to evaluate

267 the transcriptional activity of endogenous $M Y C$. Ideally, a large region of the $M Y C$ promoter on

268 Chr.8q24 would be applied to better understand the full $M Y C$ promoter mechanism. However,

269 even short region on the $M Y C$ promoter region are still not fully understood. In designing this

270 new vector, $3.1 \mathrm{~kb}$ of the promoter region was applied because of the limited length of effective

271 DNA insertion into the vector. Future improved experiments on larger region will help to clarify

272 the whole mechanism of $M Y C$ regulation via the promoter region.

273 By applying this tool to the CRISPR library, we screened molecules that increase the

274 expression of endogenous $M Y C$. According to previous reports, by introducing the CRISPR

275 library into a malignant tumor cell line and carrying out selection with a drug, molecules that

276 cause susceptibility or resistance to that drug can be identified (Konermann et al., 2015; Gilbert

277 et al., 2014; Kampmann, 2018; Kurata et al., 2018; Shalem et al., 2014; Wang et al., 2014; 
278 Koike-Yusa et al., 2014; Doench et al., 2016; Jain et al., 2016). However, this is the first specific 279 report of a molecule that activates transcription upstream of MYC.

280 MYC is an indispensable factor for cell growth and proliferation in normal tissues, but at the 281 same time, it is one of the most well-known proto-oncogenes, with high amplification in various 282 carcinomas. Moreover, MYC is widely involved in tumor development and growth (Dang, 2012).

283 284 285 286 287 288 289 290 291

Elucidation of factors that regulate the transcriptional activity of $M Y C$ is crucial for establishing new therapeutic strategies for tumors. Although $M Y C$ expression is strictly regulated by mRNA and MYC protein, in 1998, He et al. reported that inactivation of APC located downstream of Wnt signaling pathway results in excessive accumulation of $\beta$-catenin and promotes endogenous MYC expression by acting on TCF present in the promoter region of MYC.

Regarding the research of the regulatory mechanism of MYC, Vo et al. (2018) transplanted CRISPR/dCas9-VP160 and gRNAs that activated MYC transcription in the mouse brain, resulting in the development of medulloblastoma. They concluded that this model is useful for future drug research by suppressing MYC expression.

Because this screening was adapted to about 70,290 kinds of gRNAs, initially, multiple molecules were expected to be identified; however, the screening eventually led to the identification of only one molecule, MIAP. The reason for this is that, on performing single-cell collection using flow cytometry, to avoid collecting false-positive cells, the cells with the highest fluorescence intensity were collected; as a result, the number of collected cells was extremely small. Furthermore, since the proliferation of the cells after the collection was poor, it is expected that collected cells included cells that were highly brightened due to cell senescence. Moreover, oncogene-induced cell senescence can be one explanation for why the survival rate of collected single cells was very low. Oncogene-induced senescence, which is now a well-known 
301 phenomenon, causes cell growth arrest where the oncogene is excessive. This was first reported

302 in RAS (Serrano et al., 1997), and the same mechanism has been reported for MYC, where MYC

303 oncogene overexpression results in inactivation of $M Y C$, followed by the arrest of cell growth

304 and proliferation ( $W u$ et al., 2007). Excessive $M Y C$ expression due to the CRISPR activation

305 system may have induced cell senescence and caused a low survival rate of sorted cells. We

306 would like to use a next-generation sequencing system and adjust the multiplicity of infection in

307 future screenings.

308 By newly established experimental line in this study, M1AP, which was found as a transcription 309 factor for $M Y C$, was proved to function strongly as a $M Y C$ activator by promoter assay. On the 310 other hand, $M Y C$ expression was mildly elevated in the $M 1 A P$-overexpressing cells collected at 311 the same time as the luciferase assay. These results suggest that the $M Y C \mathrm{mRNA}$ is complexly 312 controlled by multiple factors and multiple pathways.

313 In conclusion, the present study confirmed that this experimental method applying the CRISPR 314 library technology functioned effectively for the identification of the molecule that activates 315 endogenous $M Y C$. This method will help elucidate the regulatory mechanism of $M Y C$ expression, 316 as well as facilitating further drug research against malignant tumors. However, more 317 investigation is required to establish a more accurate and precise screening technique. 318 319 320 321 322 323 


\section{Acknowledgments}

325 The authors would like to thank tor the member of stem cell laboratory Tokyo Medical and 326 Dental University, for cell sorting and her technical assistance and advice.

327

328

329

330

331

332

333

334

335

337

338

339

340

341

342

343

344

345

346

\section{References}

Bagger FO, Sasivarevic D, Sohi SH, Laursen LG, Pundhir S, Sonderby CK, Winther O, Rapin N, Porse BT. 2016. BloodSpot: a database of gene expression profiles and transcriptional programs for healthy and malignant haematopoiesis. Nucleic acids research, 44:D917-24.

Cancer Genome Atlas Research Network, Weinstein JN, Collisson EA, Mills GB, Shaw KR, Ozenberger BA, Ellrott K, Shmulevich I, Sander C, Stuart JM. 2013. The Cancer Genome Atlas Pan-Cancer analysis project. Nature genetics, 45:1113-1120.

Cerami E, Gao J, Dogrusoz U, Gross BE, Sumer SO, Aksoy BA, Jacobsen A, Byrne CJ, Heuer ML, Larsson E, Antipin Y, Reva B, Goldberg AP, Sander C, Schultz N. 2012. The cBio cancer genomics portal: an open platform for exploring multidimensional cancer genomics data. Cancer discovery, 2:401-404.

Dang CV. 2012. MYC on the path to cancer. Cell, 149:22-35.

Doench JG, Fusi N, Sullender M, Hegde M, Vaimberg EW, Donovan KF, Smith I, Tothova Z, Wilen C, Orchard R, Virgin HW, Listgarten J, Root DE. 2016. Optimized sgRNA design to maximize activity and minimize off-target effects of CRISPR-Cas9. Nature biotechnology, 34:184-191.

Gao J, Aksoy BA, Dogrusoz U, Dresdner G, Gross B, Sumer SO, Sun Y, Jacobsen A, Sinha R, Larsson E, Cerami E, Sander C, Schultz N. 2013. Integrative analysis of complex cancer genomics and clinical profiles using the cBioPortal. Science signaling, 6:pl1. 
347 Gilbert LA, Horlbeck MA, Adamson B, Villalta JE, Chen Y, Whitehead EH, Guimaraes C,

348 Panning B, Ploegh HL, Bassik MC, Qi LS, Kampmann M, Weissman JS. 2014. Genome-Scale

349 CRISPR-Mediated Control of Gene Repression and Activation. Cell, 159:647-661.

350 Jain IH, Zazzeron L, Goli R, Alexa K, Schatzman-Bone S, Dhillon H, Goldberger O, Peng J,

351 Shalem O, Sanjana NE, Zhang F, Goessling W, Zapol WM, Mootha VK. 2016. Hypoxia as a

352 therapy for mitochondrial disease. Science (New York, N.Y.), 352:54-61.

353 Kampmann M. 2018. CRISPRi and CRISPRa Screens in Mammalian Cells for Precision Biology

354 and Medicine. ACS chemical biology, 13:406-416.

355 Koike-Yusa H, Li Y, Tan EP, Velasco-Herrera Mdel C, Yusa K. 2014. Genome-wide recessive

356 genetic screening in mammalian cells with a lentiviral CRISPR-guide RNA library. Nature

357 biotechnology, 32:267-273.

358 Konermann S, Brigham MD, Trevino AE, Joung J, Abudayyeh OO, Barcena C, Hsu PD, Habib

359 N, Gootenberg JS, Nishimasu H, Nureki O, Zhang F. 2015. Genome-scale transcriptional

360 activation by an engineered CRISPR-Cas9 complex. Nature, 517:583-588.

361 Kong YW, Cannell IG, de Moor CH, Hill K, Garside PG, Hamilton TL, Meijer HA, Dobbyn HC,

362 Stoneley M, Spriggs KA, Willis AE, Bushell M. 2008. The mechanism of micro-RNA-

363 mediated translation repression is determined by the promoter of the target gene. Proceedings

364 of the National Academy of Sciences of the United States of America, 105:8866-8871.

365 Kurata M, Rathe SK, Bailey NJ, Aumann NK, Jones JM, Veldhuijzen GW, Moriarity BS,

366 Largaespada DA. 2016. Using genome-wide CRISPR library screening with library resistant

367 DCK to find new sources of Ara-C drug resistance in AML. Scientific reports, 6:36199. 
368 Kurata M, Wolf NK, Lahr WS, Weg MT, Kluesner MG, Lee S, Hui K, Shiraiwa M, Webber BR, 369 Moriarity BS. 2018. Highly multiplexed genome engineering using CRISPR/Cas9 gRNA $370 \quad$ arrays. PloS one, 13:e0198714.

371 Kurata M, Yamamoto K, Moriarity BS, Kitagawa M, Largaespada DA. 2018. CRISPR/Cas9

372 library screening for drug target discovery. Journal of human genetics, 63:179-186.

373 Liu C, Li Y, Semenov M, Han C, Baeg GH, Tan Y, Zhang Z, Lin X, He X. 2002. Control of 374 beta-catenin phosphorylation/degradation by a dual-kinase mechanism. Cell, 108:837-847. 375 Novak A, Dedhar S. 1999. Signaling through beta-catenin and Lef/Tcf. Cellular and molecular $376 \quad$ life sciences : CMLS, 56:523-537.

377 Sampson VB, Rong NH, Han J, Yang Q, Aris V, Soteropoulos P, Petrelli NJ, Dunn SP, Krueger 378 LJ. 2007. MicroRNA let-7a down-regulates MYC and reverts MYC-induced growth in Burkitt 379 lymphoma cells. Cancer research, 67:9762-9770.

380 Serrano M, Lin AW, McCurrach ME, Beach D, Lowe SW. 1997. Oncogenic ras provokes 381 premature cell senescence associated with accumulation of p53 and p16INK4a. Cell, 88:593382602.

383 Shalem O, Sanjana NE, Hartenian E, Shi X, Scott DA, Mikkelson T, Heckl D, Ebert BL, Root 384 DE, Doench JG, Zhang F. 2014. Genome-scale CRISPR-Cas9 knockout screening in human 385 cells. Science (New York, N.Y.), 343:84-87.

386 Tang Z, Li C, Kang B, Gao G, Li C, Zhang Z. 2017. GEPIA: a web server for cancer and normal 387 gene expression profiling and interactive analyses. Nucleic acids research, 45:W98-W102. 388 Tseng YY, Moriarity BS, Gong W, Akiyama R, Tiwari A, Kawakami H, Ronning P, Reuland B, 389 Guenther K, Beadnell TC, Essig J, Otto GM, O'Sullivan MG, Largaespada DA, Schwertfeger 
390 KL, Marahrens Y, Kawakami Y, Bagchi A. 2014. PVT1 dependence in cancer with MYC 391 copy-number increase. Nature, 512:82-86.

392 Vo BT, Kwon JA, Li C, Finkelstein D, Xu B, Orr BA, Sherr CJ, Roussel MF. 2018. Mouse 393 medulloblastoma driven by CRISPR activation of cellular Myc. Scientific reports, 8:8733-018.

394 Wang T, Wei JJ, Sabatini DM, Lander ES. 2014. Genetic screens in human cells using the 395 CRISPR-Cas9 system. Science (New York, N.Y.), 343:80-84.

396 Wu CH, van Riggelen J, Yetil A, Fan AC, Bachireddy P, Felsher DW. 2007. Cellular senescence 397 is an important mechanism of tumor regression upon c-Myc inactivation. Proceedings of the $398 \quad$ National Academy of Sciences of the United States of America, 104:13028-13033.

399

400 Figure Legends

401

402 Figure 1. A scheme for MYC transcriptional activator screening with the CRISPR 403 activation library.

404

405 Figure 2. Dendra2-positive cells using fluorescence microscope.

406 (A)-(L) correspond Clone 1-12. pMYC-promoter-Dendra2 was transfected into 12 clones that 407 were successfully cloned after CRISPR activation screening. Clones 3 and 5 showed Dendra2 408 positivity.

409

410 Figure 3. Validation experiments for candidate genes as $M Y C$ activators.

411 (A) PLEKHO2 (B) MICU3 (C) MBTPSI (D) MIAP

412 With gRNAs of the four nominated genes, $\mathrm{p} M Y C$-promoter-Dendra2 was transfected into HEK $413293 \mathrm{~T}$ cells by applying the CRISPR activation system. Only MIAP-introduced cells were 414 Dendra2 positive.

415 
416 Figure 4. $M Y C$ mRNA expression analyzed by real-time quantitative polymerase chain 417 reaction.

418 (A) Vector map of Dox-inducible $M 1 A P$ expression vector. (B) High $M Y C$ expression was 419 observed in DOX-induced cells ( $t$-test, $p<0.05)$. (C) Western blotting analysis showed 420 increasing of MYC protein in DOX- induced cells. (D) Luciferase activity at 48 hours was 421 significantly higher in $M 1 A P$-overexpressing cells ( $t$-test, $p<0.05)$. (E) $M Y C$ mRNA expression 422 at 48 hours was mildly higher in M1AP-overexpressing cells ( $t$-test, $p<0.05)$.

423

424 Figure 5. Correlation between $M Y C$ and $M 1 A P$ in the database.

425 (A) In cBioPortal using TCGA data, a correlation analysis between $M Y C$ and MIAP in AML 426 revealed a mild positive correlation.

427 (B) Using the Bloodspot database M1AP mRNA expression in acute myeloid leukemia (AML, $428 \mathrm{~N}=989$ ) was significantly higher than in normal tissue ( $\mathrm{N}=397)$. (Mann-Whitney $\mathrm{U}$ test, $p<$ $4290.05)$

430 (C) The BloodSpot database was used to retrieve gene expression data. M1AP expression was 431 higher in each subtype of AML, especially with t (8;21). (Kruskal-Wallis test, *: $p<0.01$ ) 432

Table 1. List of Candidate Genes for MYC Activation

434

435

436

437

438

439

440

441

442

Supplementary Figures

Fig. 1S. Construct of $M Y C$ promoter-reporter system.

Fig. 2S. Photoswitchable attribute of Dendra2.

Fig. 3S. Luciferase activity in $M 1 A P$-overexpressing cells and $M Y C$ mRNA expression at 72

443

hours. $M Y C$ promoters are strongly activated at 72 hours after induction of MIAP expression, 444

445 but the mRNA of MYC was not significantly upregulated at 72 hours.

Fig. 4S. $M 1 A P$ expression and correlation with $M Y C$ in databases.

(A) Comparison of $M 1 A P$ expression in normal and tumor tissue in GEPIA. 
447 (B)-(F) Correlations between $M Y C$ and $M 1 A P$ in cBioPortal.

448 (B) Cervical carcinoma. (C) Ovarian carcinoma. (D) Thyroid carcinoma. (E) Uterine endometrial 449 carcinoma. (F) Uterine carcinosarcoma.

450 
Table $\mathbf{1}$ (on next page)

List of Candidate Genes for MYC Activation 


\section{Table 1}

2

\begin{tabular}{cc} 
Sequence of gRNA & Gene name \\
\hline GGGCGGGAAATGGGTGGGGA & PLEKHO2 \\
TTGTGACACTACTCCAGCCT & MICU1 \\
AGGATCCCCGAAAAGGAGCA & $M B T P S 1$ \\
TCTCAGGGTATCTAGGGACT & $M 1 A P$
\end{tabular}

3 
Figure 1

A scheme for MYC transcriptional activator screening with the CRISPR activation library.

\section{HEK293T}

$\left(1 \times 10^{7}\right.$ cells $)$

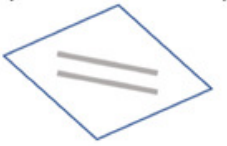

pLenti-MS2-P65-HSF1-Hygromycin pLenti-dCas9/VP64-Blasticidin

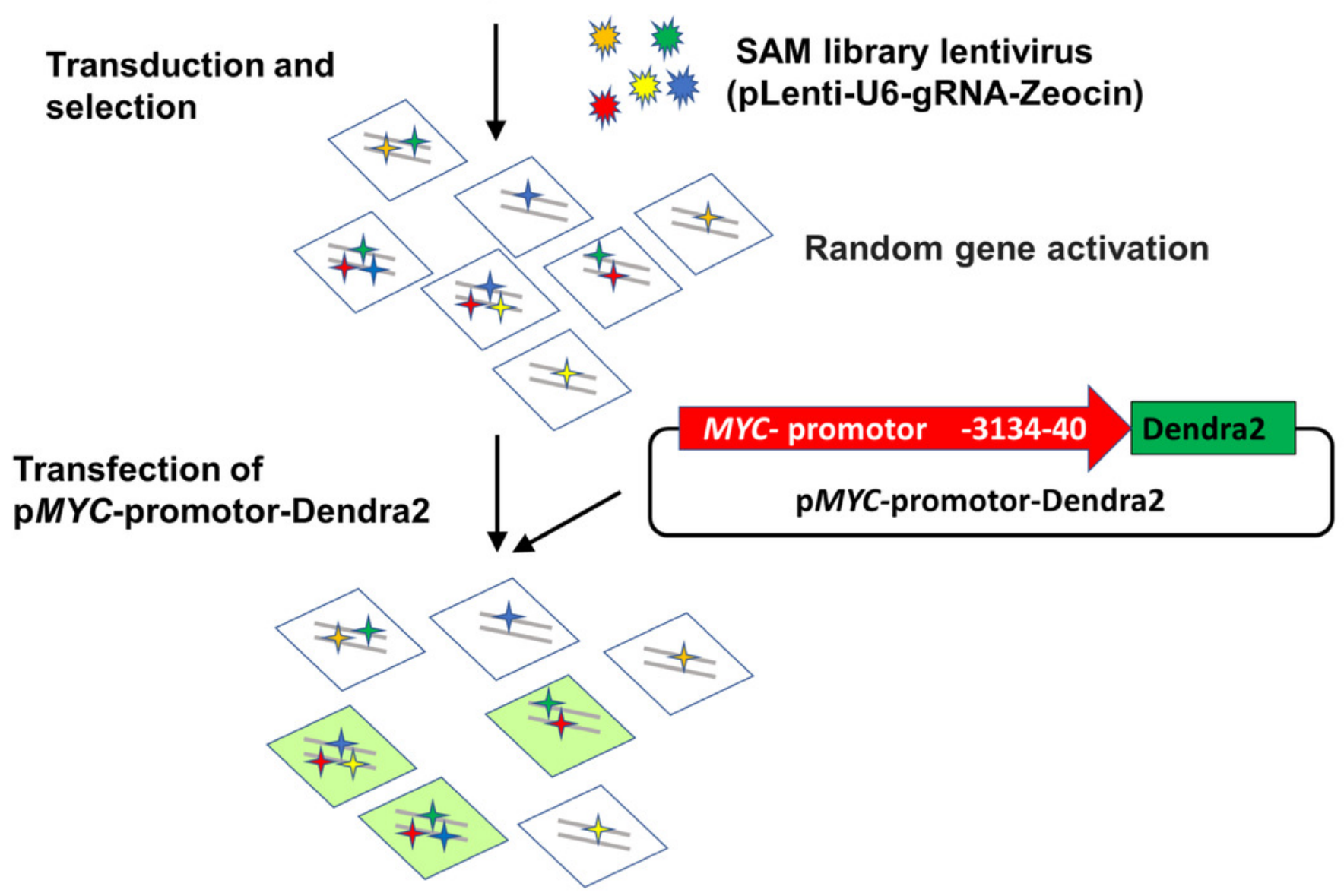

Collection of Dendra2-

positive cells by FCM into

96well plate

(positive ratio:0.017\%) 
Figure 2

Dendra2-positive cells using fluorescence microscope.

(A)-(L) correspond Clone 1-12. p MYC- promoter-Dendra2 was transfected into 12 clones that were successfully cloned after CRISPR activation screening. Clones 3 and 5 showed Dendra2 positivity. 


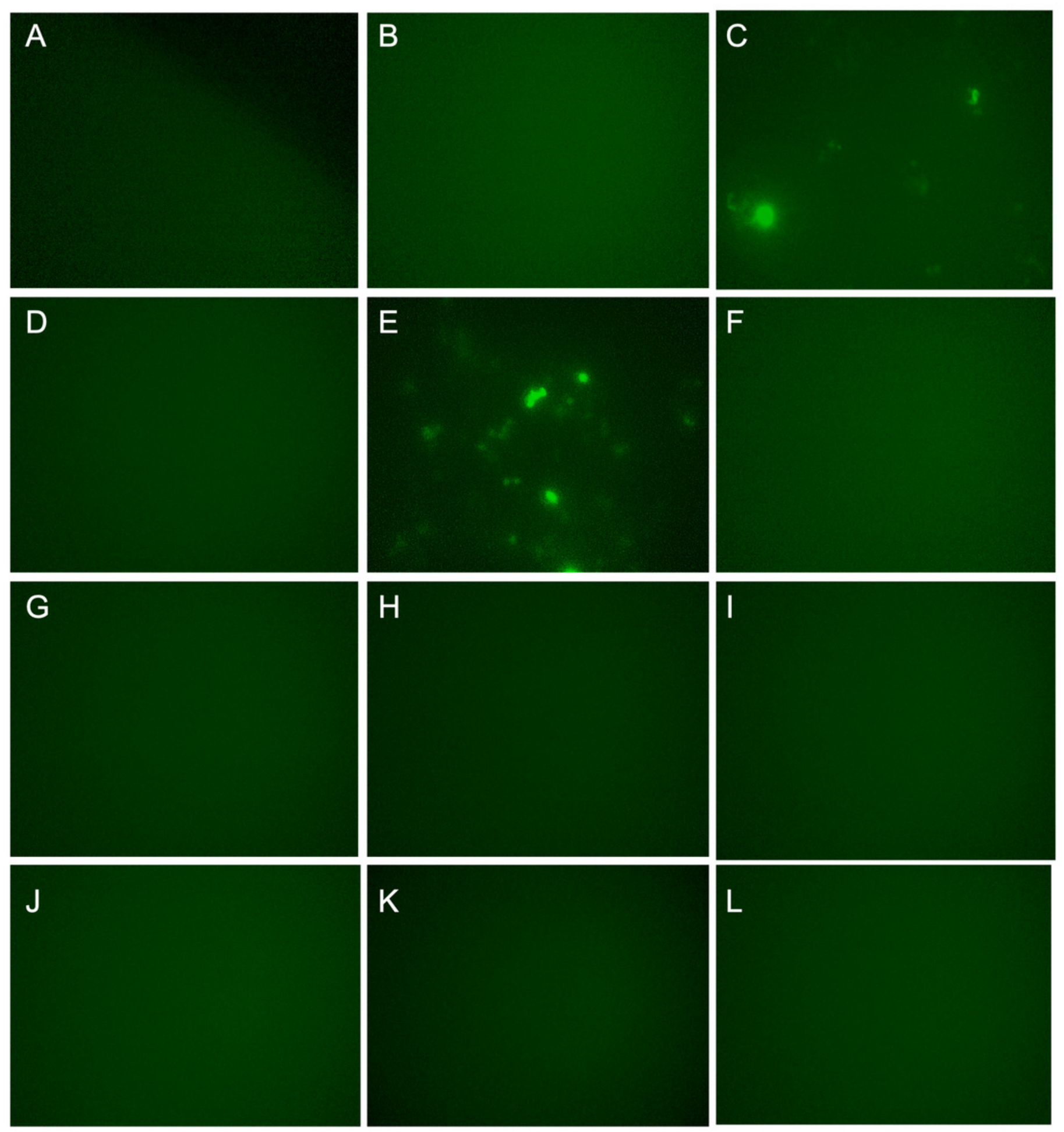


Figure 3

Validation experiments for candidate genes as MYC activators

(A) PLEKHO2 (B) MICU3 (C) MBTPS1 (D) M1AP With gRNAs of the four nominated genes, $p$ MYC- promoter-Dendra2 was transfected into HEK 293T cells by applying the CRISPR activation system. Only M1AP-introduced cells were Dendra2 positive. 


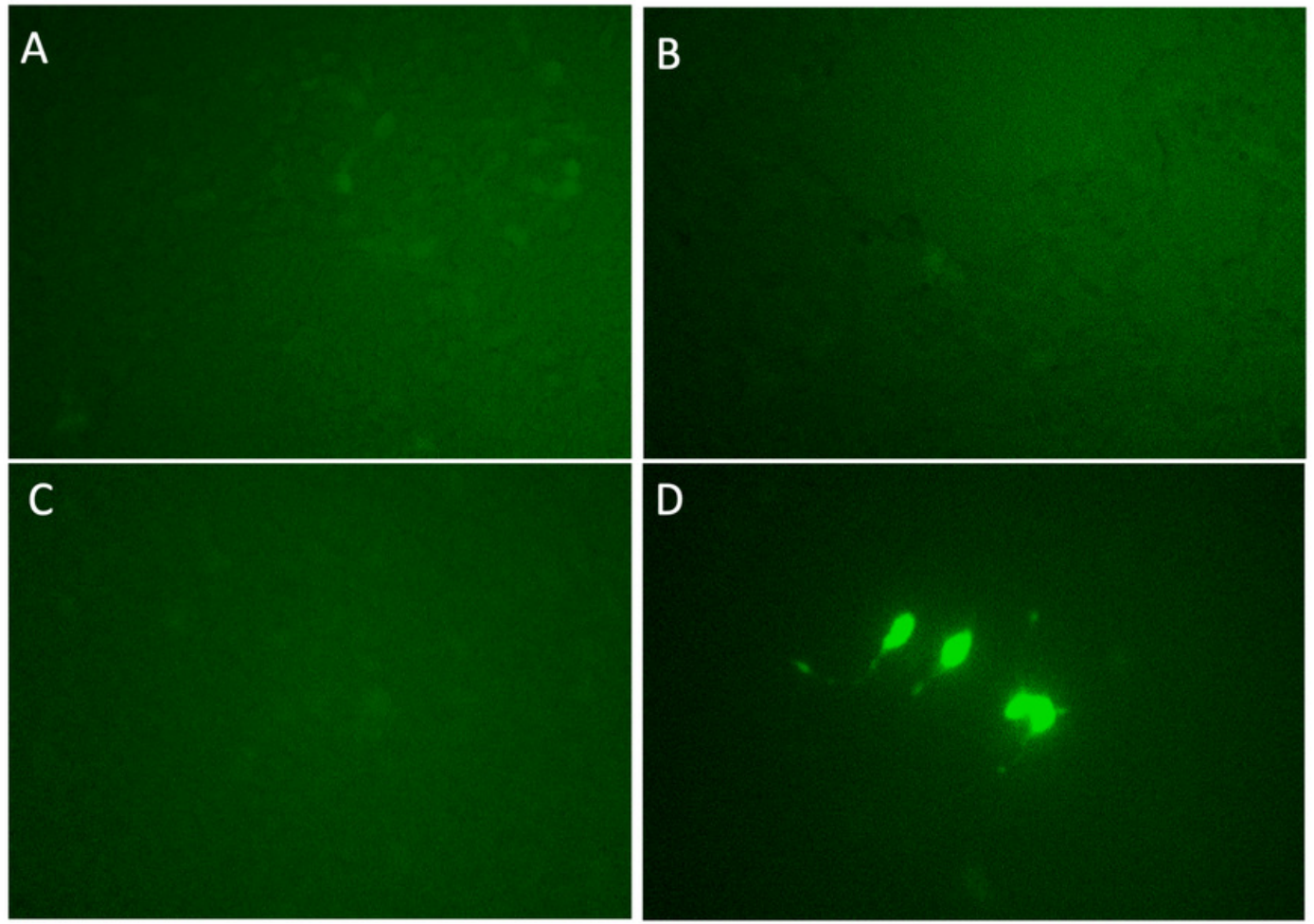




\section{Figure 4}

MYC mRNA expression analyzed by real-time quantitative polymerase chain reaction.

(A) Vector map of Dox-inducible M1AP expression vector. (B) High MYC expression was observed in DOX-induced cells ( $t$-test, $p<0.05$ ). (C) Western blotting analysis showed increasing of MYC protein in DOX- induced cells. (D) Luciferase activity at 48 hours was significantly higher in M1AP-overexpressing cells ( $t$-test, $p<0.05$ ). (E) MYC mRNA expression at 48 hours was mildly higher in M1AP-overexpressing cells (t-test, $p<0.05$ ). 
A
LTR
TRE
M1AP
IRES
GFP $E F 1 \alpha$
rtTA
Puro
LTR
PTRIPZ-M1AP-GFP

B

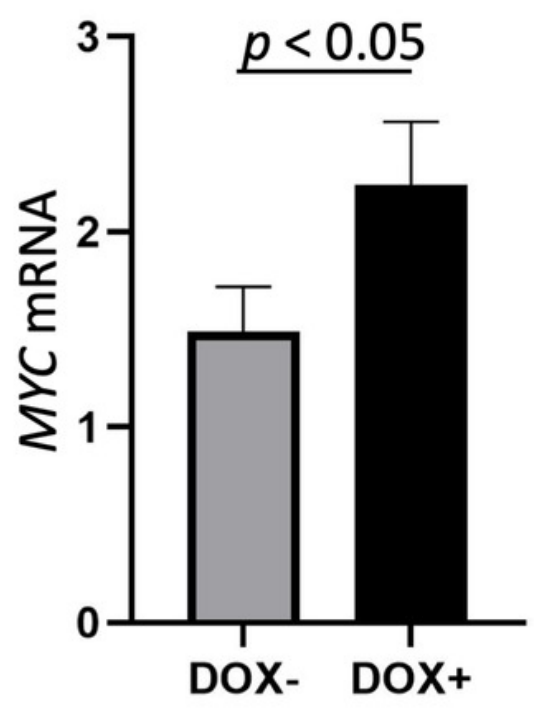

D

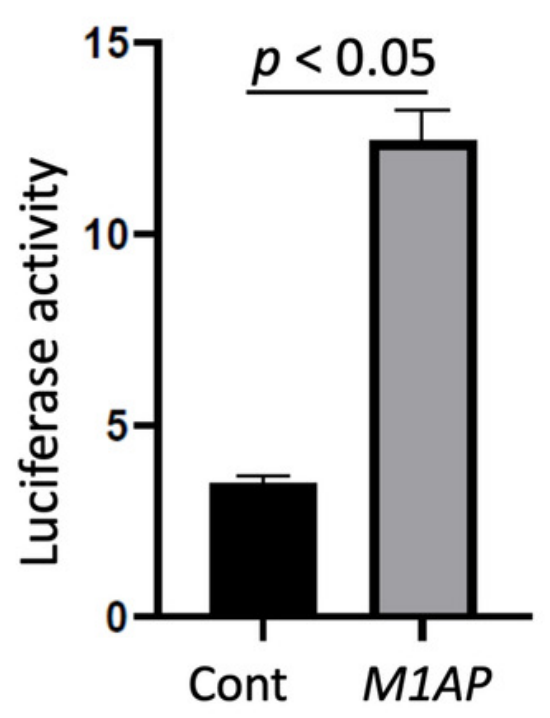

C

\section{DOX- DOX+}

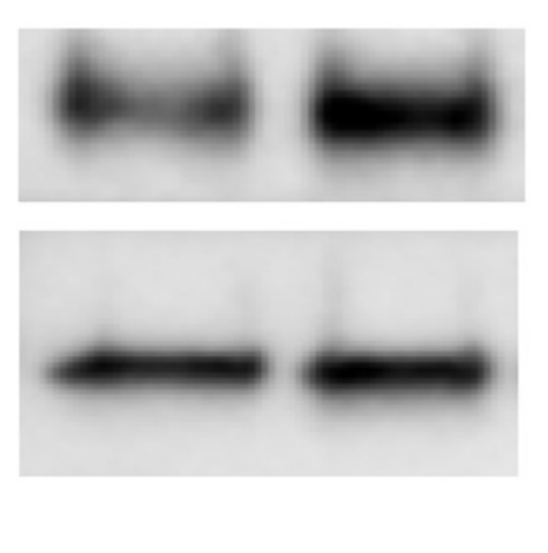

MYC

GAPDH

E

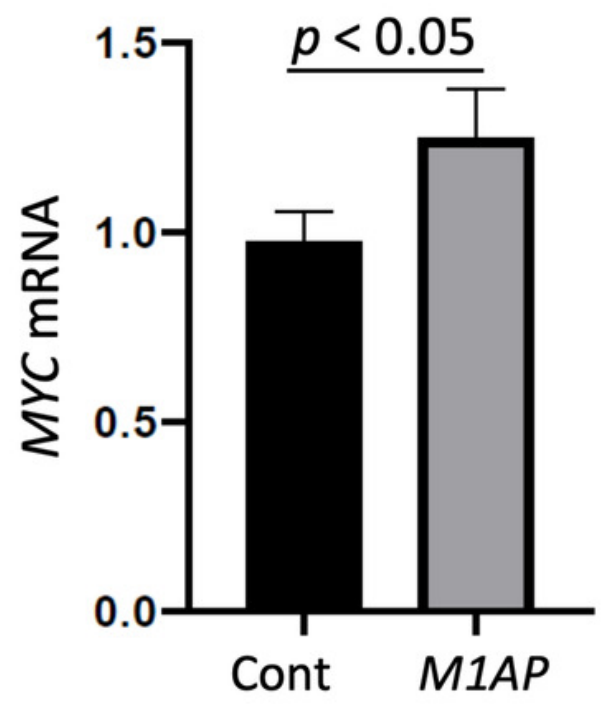




\section{Figure 5}

Correlation between MYC and MIAP in the database.

(A) In CBioPortal using TCGA data, a correlation analysis between MYC and MIAP in AML revealed a mild positive correlation. (B) Using the Bloodspot database M1AP mRNA expression in acute myeloid leukemia ( $A M L, N=989$ ) was significantly higher than in normal tissue ( $N=397$ ). (Mann-Whitney $\mathrm{U}$ test, $p<0.05$ ) (C) The BloodSpot database was used to retrieve gene expression data. M1AP expression was higher in each subtype of AML, especially with $\mathrm{t}(8 ; 21)$. (Kruskal-Wallis test, $*: p<0.01$ ) 


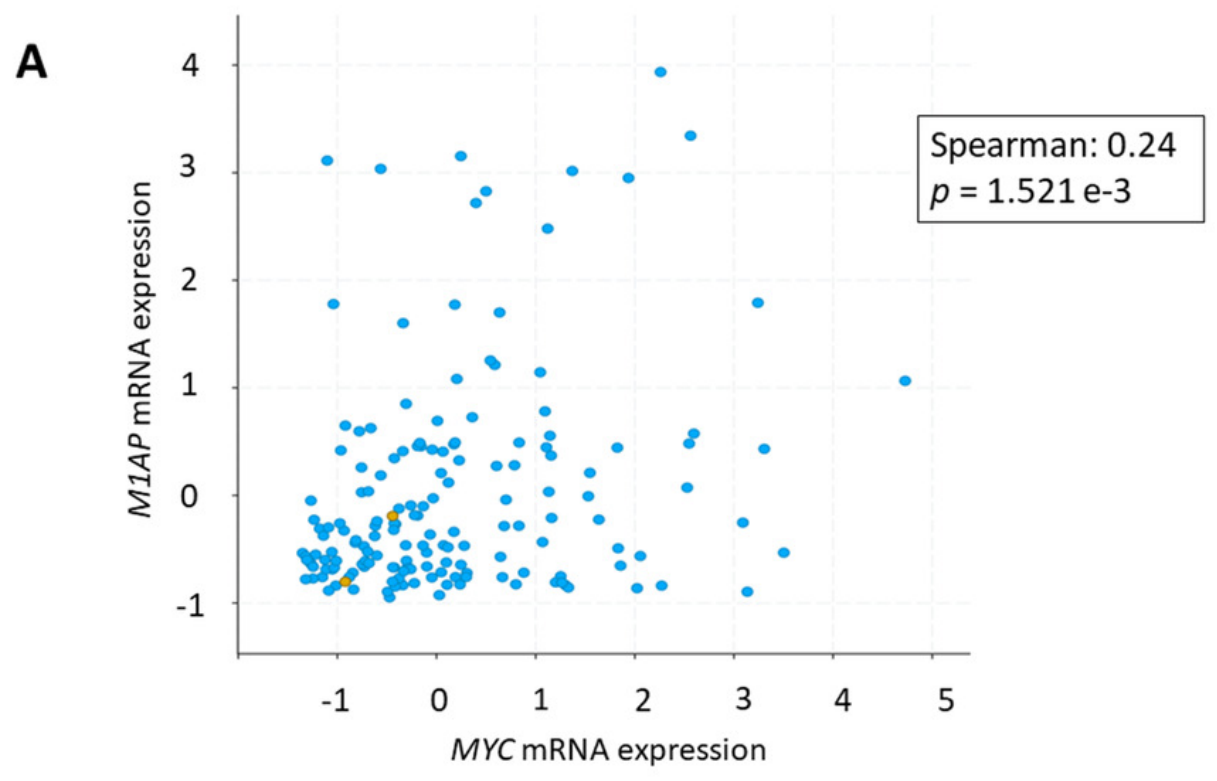

B

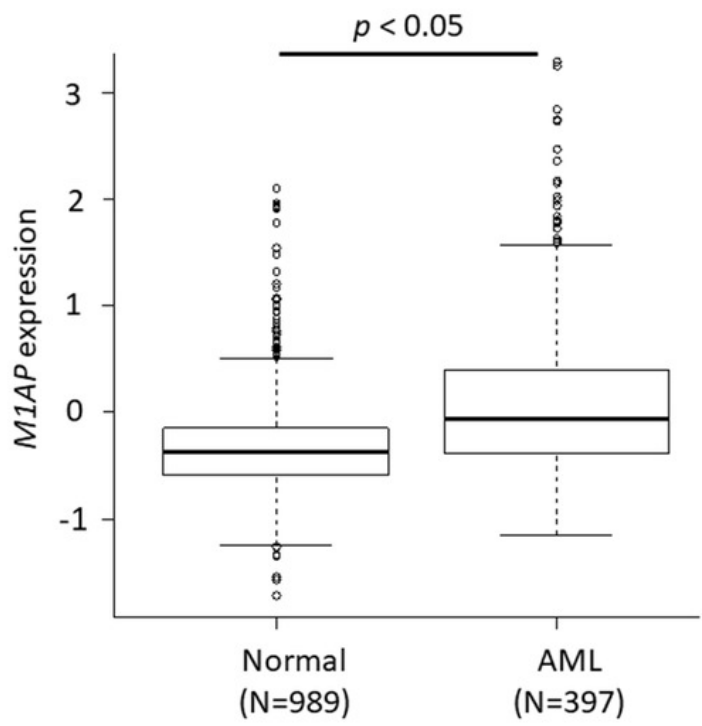

C

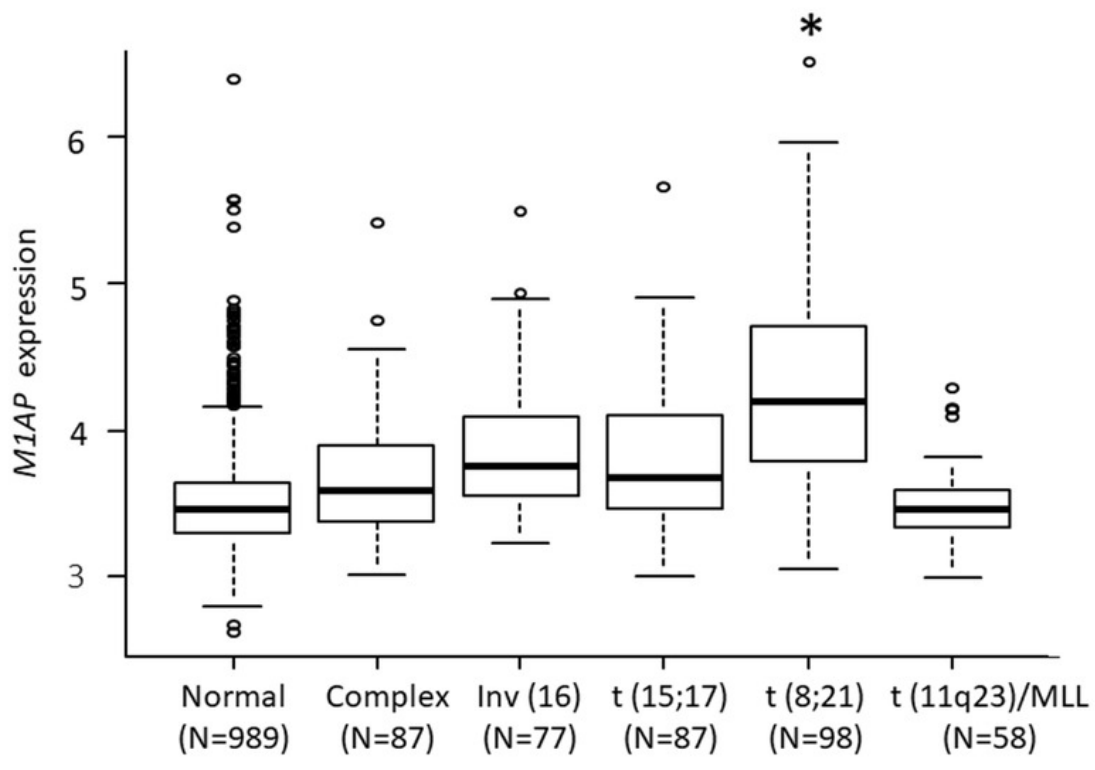

Peer) reviewing PDF | (2019:12:44132:1:1:NEW 18 Mar 2020) 